\title{
La industria 4.0, el nuevo motor de la innovación industrial
}

\author{
Xavier Ayneto Gubert
}

Recibido: 30 de Mayo de 2019 / Aceptado: 1 de Septiembre de 2019

\section{Resumen}

La industria afronta el gran reto de una nueva revolución. No se trata solo de la digitalización de los procesos existentes, si no de un cambio profundo de modelo hacia una fabricación personalizada, adaptativa, descentralizada, flexible, eficiente y en masa.

A la hibridación entre los mundos físico y virtual, que interactúan en tiempo real a través de sistemas ciberfísicos, se une una mayor integración y nuevos modelos de negocio.

La innovación y la formación de nuevos profesionales con una visión transversal, alto nivel tecnológico y nuevas competencias. toman un valor central en el camino hacia este nuevo paradigma industrial.

\section{Palabras clave}

Industria 4.0, servitización, innovación, formación superior, digitalización.

\section{Introducción}

La industria afronta el gran reto de la cuarta revolución industrial. No se trata solo de la digitalización de los procesos existentes, si no de un cambio profundo de modelo hacia una fabricación personalizada, adaptativa, descentralizada, flexible, eficiente y en masa.

A la hibridación entre los mundos físico y virtual, que interactúan en tiempo real a través de los nuevos sistemas ciberfísicos, se une una mayor integración a todos los niveles: vertical, horizontal y a lo largo del ciclo de vida, así como la posibilidad de crear nuevos modelos de negocio basados en la servitización y los datos.

La innovación, tanto tecnológica como no tecnológica, toma pues un valor central en el camino hacia el nuevo paradigma de la industria 4.0 en cualquiera de sus tres grandes vías: la innovación interna, a través del intraemprendimiento, la innovación abierta en cooperación, y la innovación externa en colaboración con empresas tecnológicas y startups, creando nuevas cadenas de valor con más espacio para nuevos jugadores.

Este cambio de paradigma exige además formar nuevos profesionales de la industria con una visión transversal, alto nivel tecnológico y nuevas competencias que les permitan desenvolverse con éxito.

Xavier Ayneto Gubert *

orcid.org/0000-0003-4373-7603

* Prof.Titular RMEE. Universidad Politécnica de Catalunya
El presente artículo analiza estos aspectos en tres grandes bloques:

- $\quad$ El reto de la industria 4.0; concepto y tecnologías.

- Cómo afrontar la innovación asociada a este cambio de paradigma.

- Qué implicaciones supone dicho cambio en la formación superior de los nuevos profesionales de la industria.

\section{Una visión de conjunto}

\subsection{No es solo un tema de competitividad, que también}

El gran reto que la industria 4.0 ha venido a resolver, no es solo el de la competitividad de los países avanzados. Está aquí para dar respuesta a un mundo que cambia de forma acelerada y en el que clientes y consumidores toman, cada vez más, un papel protagonista. Se necesitan fábricas ultraflexibles, adaptables de forma continua a nuevas demandas y capaces de disfrutar de las ventajas de la economía de escala para producir productos personalizados, que además operen de forma respetuosa con el medio ambiente en un contexto de economía circular. Diversos países han tomado conciencia de ello y han definido sus propias estrategias de implementación de la industria 4.0 en sus políticas industriales [1,2,3] 


\section{2. ¿Pero cómo hemos llegado hasta aquí?}

Desde inales del siglo XVIII se han vivido al menos 3 grandes revoluciones industriales (Figura 1), ligadas esencialmente al uso de nuevas formas de energía y de nuevas tecnologías que han permitido a su vez nuevas formas de organización del trabajo. En la primera revolución las máquinas movidas por vapor substituyeron gran parte del trabajo artesanal. En la segunda revolución industrial, la energía eléctrica y los motores de combustión se unieron a la división del trabajo para dar entrada a la fabricación en serie. Durante el siglo XX la aparición de la electrónica, la informática y más tarde de los microprocesadores, facilitaron la auto- matización de la maquinaria. Y por último, desde inicios del siglo XXI, la aplicación de internet como medio de comunicación digital global está permitiendo la interconexión de sistemas inteligentes que generan e intercambian datos en tiempo real; sobre esta base se construye el concepto de industria 4.0. Este término, acuñado en Alemania en 2011 para designar la cuarta revolución industrial, ya identi icada como tal en 2005 por el MIT, describe la organización de los procesos de producción basada en tecnologías y dispositivos que se comunican de forma autónoma entre ellos a lo largo de la cadena de valor y mediante modelos virtuales computacionales.
Figura 1 Las 4 revoluciones industriales

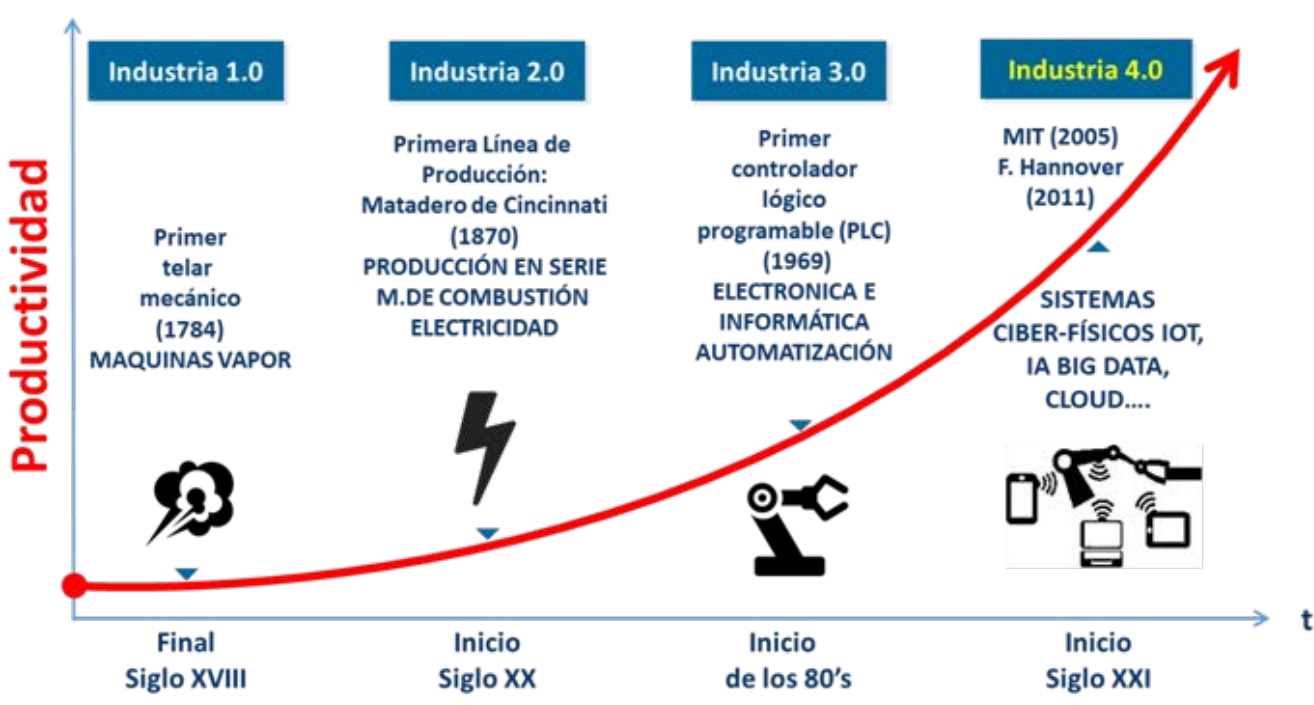

Adaptado de : Forschungsunion, acatech, Abschlussbericht Arbeitskreis Industrie 4.0

\section{3. ¿Cuáles son las características esenciales de la industria 4.0?}

Estamos en un mundo dual, con una componente física y otra digital ya desde la $3^{\circ}$ revolución industrial. En este contexto, la simulación permitió pasar del mundo físico al mundo virtual, mientras que la automatización ha facilitado controlar sistemas físicos desde modelos digitales. Pero es en esta $4^{\mathrm{a}}$ revolución que la velocidad de proceso y de intercambio de datos ha hecho emerger conceptos como la inteligencia artificial y el internet de las cosas. Los mundos virtual y real ya no son ahora dos realidades separadas sino una misma realidad dual que se concreta en la aparición de sistemas ciberfísicos (Figura2), productos y/o medios de producción, con una componente física y otra digital interconectadas, capaces de captar ingentes cantidades de datos que, debidamente tratados, ofrecen nuevas posibilidades a la organización y optimización de las operaciones de manufactura. 
Figura 2 Sistemas ciberfísicos.

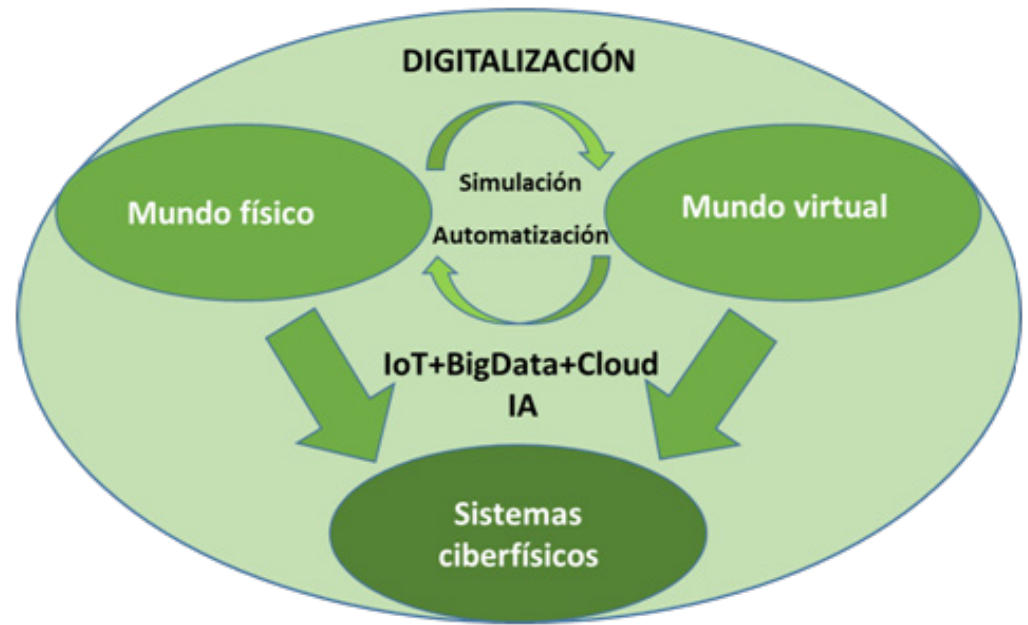

\section{4. ¿Nuevas forma de operación?}

La interconexión de dispositivos se está produciendo a una velocidad vertiginosa y a nivel global. Ahora ya no es solo posible integrar verticalmente todas las operaciones a lo largo de una cadena de valor empresarial, sino que se pueden también integrar horizontalmente y en tiempo real todas las actividades de cooperación con otras empresas a lo largo de cadenas de suministro globales. Y no solo se integran las actividades manufactureras, sino también las de ingeniería de modo que los tecnólogos de diseño, desarrollo, producción y logística interaccionan sobre un único modelo digital que engloba productos y procesos a lo largo de todo el ciclo de vida.

Las personas no son inmunes a todos estos cambios. Se necesitan ahora nuevos conocimientos y nuevas capacidades para las nuevas tareas que se generan.

\subsection{Un nuevo modelo industrial}

Así pues, la nueva industria 4.0 integra, interconecta y dota de inteligencia a elementos, muchos de los cuales ya existían anteriormente, pero que ahora interaccionan de una forma nueva en un enfoque holístico e integrador, dando lugar a un nuevo modelo industrial caracterizado por:

- Una producción personalizada, adaptativa, descentralizada, flexible, eficiente y en masa.

- $\quad$ La hibridación entre el mundo físico y el mundo virtual, que interaccionan en tiempo real sobre sistemas ciberfísicos.

- $\quad$ La integración a todos los niveles: vertical, horizontal y temporal a lo largo del ciclo de vida.
- Nuevos modelos de negocio y cadenas de valor.

- $\quad$ La creciente servitización de la industria.

- Más espacio para los nuevos jugadores: startups, spinoffs.

- Interdisciplinariedad y alto nivel tecnológico.

- Un nuevo concepto de comercio internacional y de propiedad intelectual e industrial.

\subsection{También un nuevo modelo socioeconómi- co}

La industria 4.0, aparece en un contexto de cambio radical como consecuencia de la digitalización de la sociedad y de la economía, y convive con nuevas necesidades a nivel planetario como la sostenibilidad en un sentido amplio. Ya nos hemos ido acostumbrando a términos como smart cities, smart grids, economía circular.... La fábrica inteligente y conectada es un elemento más dentro de la nueva economía de las redes, cuyo desarrollo ha sido posible gracias a la aparición de INTERNET.

\section{Un recorrido por las tecnologías faci- litadoras}

La implementación de la industria 4.0 se apoya en un conjunto de nuevas tecnologías sin las cuales no podría existir. Pero no debe confundirse el concepto de industria 4.0 con la mera existencia de dichas tecnologías, sino que más bien consiste en la forma en que se integran e interaccionan en el contexto de una fábrica inteligente $[4,5]$. 


\subsection{Robótica}

La robótica industrial es un desarrollo vigente desde la tercera revolución industrial, sin embargo los robots están pasando, de ser meros repetidores de movimientos preprogramados que actúan en entornos protegidos, a ganar en flexibilidad, autonomía e integración con los trabajadores humanos.

Por ejemplo, Los vehículos autónomos y semiautónomos, capaces de salvar obstáculos y seguir y optimizar rutas dependiendo de cada situación concreta, ya están siendo utilizados para la optimización de la logística.

Otra rama en ascenso es la robótica colaborativa, que permite que los robots se conviertan en colaboradores y facilitadores de trabajos rutinarios de las personas interaccionando con ellas en un mismo entorno productivo.

En los últimos años se ha empezado a trabajar en un concepto que va aún más allá, los exoesqueletos, verdaderos apéndices robóticos que liberan a los humanos de realizar esfuerzos integrándose con su propio cuerpo.

Los robots ya no son instalaciones fijas, voluminosas, poco flexibles ante los cambios, que incluso condicionan el layout de la fábrica; ahora son los robots quienes se adaptan a las líneas productivas y a los cambios en los procesos, los productos o las necesidades de los trabajadores.

\subsection{Simulación}

La simulación numérica ha permitido avanzar el curso de los hechos de modo que se pueden detectar y corregir los problemas sobre prototipos virtuales antes de que existan físicamente, minimizando así los costes de adaptaciones y modificaciones. El concepto de simulación se ha ido extendiendo de los productos a los procesos hasta llegar a la fábrica en su conjunto.

La digitalización asociada a la industria 4.0 permite ahora la virtualización de casi todos sus aspectos. Es posible, por ejemplo, mejorar la ergonomía en el puesto de trabajo, la interacción de las personas con robots, materiales y herramientas, y simular todas las interacciones de los elementos del proceso de fabricación en un entorno virtual con el objetivo de optimizarlo.

\subsection{Integración vertical, horizontal y a lo largo del ciclo de vida}

La integración de los datos dentro de dichas cadenas de valor permitirá su mayor optimización y automatización a través de software de gestión ERP (Enterprise resource planning) y sistemas MES (Manufacturing execution systems), permitiendo el análisis del OEE (Overall Equipment Effectiveness) y la mejora de la eficiencia operativa de la empresa en su conjunto mediante la integración entre la información general del negocio y la de los sistemas productivos.

Por otra parte, Las plataformas PLM (Product Lifecycle Management) permitirán integrar la información de todos los componentes y productos desde el diseño inicial hasta su postventa y desmantelamiento final, facilitando la compartición de datos entre los diferentes implicados a lo largo de todo el ciclo de vida, e incorporando también la voz del cliente en la mejora de los productos y servicios.

\subsection{Industrial Internet of Things (IIoT)}

Internet ha supuesto una verdadera revolución en muchos aspectos de nuestra vida, posibilitando ahora también la interconexión de todo tipo de máquinas y dispositivos. El Iternet de las cosas es uno de los pilares básicos de la Industria 4.0, constituyendo uno de los pilares clave para el desarrollo de todo el potencial de las tecnologías relacionadas con la sensórica y los sistemas embebidos.

Tradicionalmente estas tecnologías están organizadas en una pirámide de automatización vertical en la que los sensores y dispositivos de campo, con controladores de inteligencia y autonomía limitada, alimentan un sistema de control del proceso de fabricación general. El IIoT les permite dar un gran salto cualitativo al poder comunicarse e interaccionar entre ellos, combinando situaciones centralizadas y descentralizadas de toma de decisiones.

\subsection{Ciberseguridad}

Históricamente las empresas han confiado en sistemas informáticos y digitales propios, cerrados o desconectados del exterior. Por ello, el principal desafío al que se enfrentan las empresas para la implantación de la Industria 4.0, junto con el de la gestión del talento y las personas, es el de la confianza en la seguridad de los sistemas digitales dada la necesidad de garantizar la protección de los sistemas industriales críticos.

Los sistemas de Ciberseguridad permiten el control ante eventos indeseados, errores, ciberataques, o manipulaciones internas malintencionadas. Para ello se debe identificar qué es necesario proteger y con qué grado de prioridad y criticidad, monitorizar las actividades en tiempo real en modo de inspección y vigilancia de los diferentes sucesos, y aplicar las adecuadas políticas de seguridad y gestión de alertas.

\subsection{La nube industrial (Cloud)}

Ya no es necesario operar y gestionar todos los sistemas in situ, muchas empresas se han decidido ya a utilizar software basado en la nube. El cloud industrial debe permitir además 
la interconexión entre todos los dispositivos de la empresa, con flujos de datos multidireccionales de los elementos que se conectan (dispositivos móviles, maquinas, robots, software...) y es la base para que funcione y se desarrolle en todo su potencial la servitización de la industria, basada principalmente en la digitalización de sus productos, procesos y servicios.

\subsection{Fabricación aditiva (AM)}

Las empresas han empezado a usar la impresión en 3D para la fabricación de prototipos y componentes individuales. El enorme potencial de los diferentes sistemas y tecnologías de fabricación avanzada permitirá conseguir piezas más complejas y con características que no sería posible conseguir de ninguna otra forma.

Entre las principales ventajas de la fabricación aditiva se encuentran: la integración funcional, reduciendo el número de componentes y uniones en un conjunto, la personalización y optimización de los productos a niveles antes inimaginables, la disposición de sistemas de producción descentralizada que permitirá reducir costes de transporte y logístico, o la fabricación en lotes de fabricación muy cortos, incluso piezas únicas como por ejemplo los recambios, evitando stocks y permitiendo la fabricación in situ y just in time del recambio.

\subsection{Realidad virtual (VR) y aumentada (AR)}

Ya existen aplicaciones industriales de la realidad aumentada en diferentes campos. Unas de las de mayor recorrido son del diseño de producto, el mantenimiento de equipos y la formación de los trabajadores. En el primer caso, permite por ejemplo visualizar el producto e incluso interaccionar con él antes de que exista utilizando modelos virtuales. En el segundo caso permite el reconocimiento en 3D de las piezas, y acceder a los manuales de operación o mantenimiento a pie del propio. En el tercer caso, se pueden optimizar los costes de formación, y reducir al mismo tiempo las posibles consecuencias de errores causados por personal formándose in situ en fábrica.

\subsection{Big data}

Incorporar el análisis de grandes conjuntos de datos (Big Data) en el mundo industrial, permite optimizar la calidad de la producción, mejorar en eficiencia energética y en el servicio de los equipos, mejorar el rendimiento y control de la operación, reducir los costes operativos, y en definitiva mejorar la eficiencia y efectividad de las operaciones.

La recopilación y análisis de datos de muy diversas fuentes (no solo de máquinas), es una herramienta de crucial ayuda en el proceso de toma de decisiones.

\subsection{Inteligencia artificial (AI)}

En el ámbito del trabajo manual hace ya mucho que las máquinas han substituido a los humanos con ventaja. El ámbito del trabajo intelectual no escapa a esta tendencia y el proceso de substitución ha empezado ya con fuerza.

El concepto de inteligencia artificial existe desde hace décadas, pero no ha sido hasta hace pocos años que ha tomado una especial relevancia. No obstante, se está aún lejos de una inteligencia artificial robusta, aquella que emularía el razonamiento humano, y más aún de la conciencia artificial. La inteligencia artificial aplicada de que hoy disponemos puede ser de gran ayuda en la identificación de patrones y en la propuesta de soluciones a determinados problemas. El denominado machine learning, y especialmente su versión más avanzada, el deeplearning, resultan altamente eficientes en este propósito, permitiendo un creciente número de aplicaciones en diversos campos [6].

Por el momento la inteligencia artificial no puede substituir a la humana pero puede resultar de gran ayuda en el análisis de grandes volúmenes de datos y en la búsqueda de tendencias como apoyo a la toma de decisiones; sin embargo, la última decisión sigue siendo responsabilidad humana.

En la figura 3 se muestra, a modo de resumen, una posible clusterización de las diversas tecnologías diferenciales implicadas en la industria 4.0 
Figura 3 Clusterización de las tecnologías de la industria 4.0
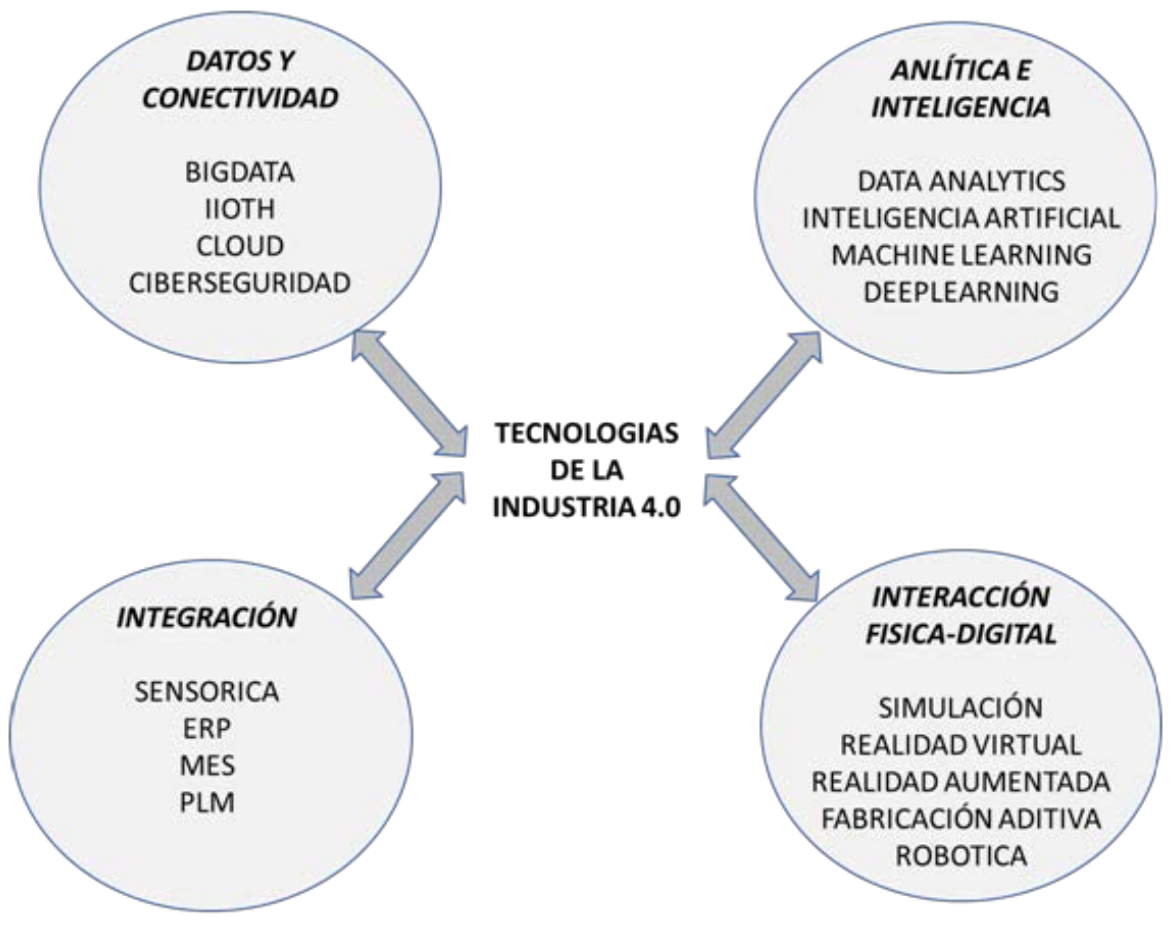

\section{4. ¿Cómo innovar en el camino hacia la industria 4.0?}

La mera exposición del gran abanico de tecnologías disponibles, impresión 3D, Big data, Cloud, IoT, etc. puede desconcertar, o incluso paralizar a más de un empresario que finalmente se autoconvenza de que esto no va con él. Nada más lejos de la realidad. La apuesta por las tecnologías de la industria 4.0, es la apuesta por la competitividad, y toda industria puede pasar a ser, total o parcialmente, una Industria 4.0 integrando de forma inteligente todas o parte de las tecnologías presentadas.

Las empresas que decidan emprender el camino hacia el nuevo paradigma de la industria 4.0, deberán integrar la innovación como un proceso estratégico de negocio y gestionarlo como tal. De ese modo pasarán de ser empresas que innovan a ser empresas innovadoras, de la innovación casual a la innovación sistemática que exige el diseño estratégico e implementación de una hoja de ruta para la industria 4.0. Pero no solo eso, también deberá desarrollar un marco organizativo y de gestión adecuados para que los innovadores internos (intraemprendedores) puedan desarrollar sus capacidades y proyectos.

El primer paso es pues establecer una estrategia 4.0 bien orientada y realista [7]. ¿Cuál es el punto de partida y cuál el objetivo a alcanzar? Conocer también qué hacen los que ya están en el camino y lo están haciendo bien a través del bechmarking [8], y finalmente definir y priorizar acciones para alcanzar sus objetivos.

Es evidente que no todas las empresas parten del mismo punto, y que tampoco precisan alcanzar todas el mismo ni- vel de implantación de la industria 4.0. Cada una debe tomar conciencia de la situación de partida y dirigirse hacia una nueva situación que le aporte valor en un sentido amplio. No se trata de adherirse a "la nueva moda" sino de actuar a medida de las necesidades sentidas, y de la evolución del entorno competitivo.

\subsection{Diagnóstico}

En ese sentido, se han desarrollado diversos esquemas de autoevaluacíón y diagnóstico. A continuación se presenta de forma breve el que propone la VDMA (Asociación Alemana de Fabricantes de Maquinaria), que a través de la Fundación Impulso [9], ha desarrollado un esquema que permite a las empresas evaluar su nivel de madurez respecto al grado de implantación de la industria 4.0, y al tiempo tomar conciencia de cuáles deben ser sus metas en este campo.

\section{El análisis se realiza desde 6 puntos de vista.}

Estrategia y organización: ¿Hasta qué punto se ha integrado e implementado el modelo de Industria 4.0 en la estrategia de la empresa?

Industria 4.0 es mucho más que digitalización. Es una oportunidad para desarrollar nuevos modelos de negocio. Pero para su implantación es esencial desarrollar una cultura y estrategia adecuadas y ello depende, en gran medida, de un compromiso inequívoco de la alta dirección que asegure la asignación y aplicación eficiente de los recursos necesarios. Algunos de estos nuevos modelos de negocio entroncan, directamente y de forma natural, con el otro gran paradigma de la economía circular [10]. 
Fábrica inteligente: ¿En qué medida la empresa tiene una producción digitalmente integrada y automatizada basada en sistemas ciberfísicos?

En una primera fase, unidades de trabajo inteligentes controlarán y supervisarán el proceso de producción. Más adelante, se guiarán de manera autónoma a través de la producción. Los sistemas ciberfísicos, enlazan los mundos físico y virtual mediante la IIoT y a través de la recopilación, almacenamiento y procesamiento de datos.

Operaciones inteligentes: ¿Cuál es el nivel de Integración vertical, en toda la empresa, y horizontal, entre empresas colaboradoras en sus dimensiones física y virtual?

La digitalización y la gran cantidad de datos que ha aportado a la producción y la logística, permitirán en el futuro formas y enfoques nuevos en los sistemas de planificación de la producción y gestión de la cadena de suministro.

Productos inteligentes: ¿Los productos físicos están equipados con componentes TIC capaces de recopilar datos sobre su entorno y su estado, y comunicarse e interactuar con sistemas de alto nivel a lo largo de la cadena de valor?

En el futuro los productos conocerán su itinerario a través de la cadena de producción y se comunicarán con los diversos sistemas industriales. Los procesos podrán ser mejorados y guiados de forma autónoma y en tiempo real.

Servitización (servicios basados en datos): ¿Hasta qué punto los servicios ofertados están basados en datos y son posibles gracias a la integración de productos, producción y clientes?

Los servicios basados en datos facilitarán nuevos modelos de negocio y mejorarán el beneficio para el cliente. El negocio de postventa y servicios se basará en la evaluación y análisis de los datos recogidos y en la integración vertical. Los productos físicos estarán equipados con sensores y IT para que puedan enviar, recibir o procesar la información [11].

Desarrollo de las personas: ¿Disponen los empleados de los conocimientos y habilidades necesarios para la industria 4.0 ?

Los empleados son los más afectados por los cambios en el lugar de trabajo digital. Su entorno de trabajo directo se alterará y ello les obligará a adquirir nuevas habilidades y cualificaciones. Hay que prepararles para estos cambios con la formación apropiada y la educación continua. Este aspecto se aborda más adelante con mayor detalle.

\subsection{Implementación}

Tras la definición de una estrategia, la empresa debe empezar su camino hacia la industria 4.0 poniendo en marcha proyectos piloto (pruebas de concepto), que le permitan experimentar, aprender rápido y a bajo coste. Cada piloto debe plantear objetivos específicos dentro de la estrategia global. Es importante asumir el carácter de aprendizaje de estos proyectos, lo que incluye también la posibilidad de fracaso. Posteriormente habrá que construir a partir de los resultados de los pilotos, y decidir las nuevas capacidades necesarias que se deberán generar o adquirir.

Dichos pilotos deben enfocarse a un conjunto de impulsores de valor claves para cada tipo de industria [4]., a fin de conseguir el éxito durante su posterior escalado a toda la organización. La falta de claridad con respecto al valor comercial, unos recursos limitados, o un excesivo número de pilotos dejan a la mayoría de las empresas atrapadas en lo que en [12] denominan el "purgatorio de los pilotos"

La complejidad de los nuevos retos hará necesario adoptar una perspectiva de ecosistema. Se tendrá que recurrir en muchos casos a la innovación abierta y a la interacción de nuevos players industriales a fin de poder ofrecer soluciones completas (bienes+servicios). Las empresas industriales deben tomar conciencia de que a menudo no disponen internamente de los conocimientos y recursos necesarios para desarrollar los proyectos destinados a materializar sus innovaciones. Deben abrir entonces el proceso de innovación de manera que el conocimiento y las tecnologías fluyan bidireccionalmente a través de sus fronteras hacia agentes externos. Más allá de esto, cuando el nivel de innovación interna resulta insuficiente, debido a que las presiones del día a día, y muchas veces su propia cultura, no le permiten asumir los riesgos ni dedicar los recursos necesarios al proceso de innovación, deberá explorar alternativas externas para las innovaciones más disruptivas, como la colaboración con empresas tecnológicas avanzadas, incluidas las startups de base tecnológica.

Como ya se ha dicho más arriba, la industria 4.0 precisará sin duda de nuevas capacidades y conocimientos.

\section{Cómo disponer de los profesionales que el desarrollo de la industria $\mathbf{4 . 0}$ precisa?}

El último gran reto es sin duda la adaptación de los profesionales actuales, y también de los programas educativos para los profesionales futuros, a los cambios que implica el nuevo paradigma de la industria 4.0. 
Como ejemplo de dicha necesidad, basta considerar el cambio que la fabricación aditiva supone no solo a nivel de medios de producción, sino también, y más importante, a nivel de la conceptualización de las nuevas partes y componentes.

Lo que antes era imposible, o muy difícil, de fabricar con los medios convencionales, ya no se consideraba como una opción valida de diseño. La fabricación aditiva ha derribado esta barrera minimizando las restricciones de diseño permitiendo, por ejemplo, procesos de optimización topológica que hasta hace poco eran meras curiosidades científicas. En cuanto a formas estructurales, ahora es posible diseñar a nivel de mesoescala estructuras con propiedades nuevas antes casi inimaginables, por ejemplo, una fácil adherencia al tejido óseo de las prótesis. La posibilidad de imprimir materiales blandos y embeber en ellos sensores supone también una revolución que permite disponer de componentes inteligentes con nuevas funcionalidades y prestaciones.

Por tanto, no solo hay que transmitir el conocimiento sobre los nuevos métodos de producción como alternativas a la fabricación con métodos convencionales, sino que también hay que abrir el abanico de posibilidades en cuanto a la conceptualización de soluciones antes imposibles. Esto significa rehacer y/o completar muchos de los enfoques aceptados hasta el momento sobre la formación especialmente de los ingenieros, como demuestran los diversos artículos publicados sobre esta cuestión [13,14,15]

Se han realizado diversos estudios analizando el impacto de la industria 4.0 sobre los conocimientos y habilidades que se requerirán para los trabajadores de la industria [16,17].. Es evidente que habrá un cambio tanto en la tipología como en el nivel de las mismas. Es cierto que las fábricas del futuro requerirán menos personal de baja cualificación, pero no es menos cierto, que se abrirán nuevas oportunidades laborales de alta cualificación asociadas a las nuevas tecnologías, como la robótica, la IIoT, la IA etc.

En el ámbito más general de las habilidades personales demandadas y sus prioridades, se está produciendo un cambio substancial, tal como se refleja de forma resumida en la tabla 1 para las 10 más relevantes [16]:

Tabla 1 Las 10 habilidades principales según el World Economic Forum

\begin{tabular}{|c|c|}
\hline En 2015 & En 2020 \\
\hline 1. Resolución de problemas complejos & 1. Resolución de problemas complejos \\
\hline 2. Coordinación con otros & 2. Pensamiento crítico \\
\hline 3. Gestión de personas & 3. Creatividad \\
\hline 4. Pensamiento crítico & 4. Gestión de personas \\
\hline 5. Negociación & 5. Coordinación con otros \\
\hline 6. Control de calidad & 6. Inteligencia emocional \\
\hline 7. Orientación al servicio & 7. Juicio y toma de decisiones \\
\hline 8. Juicio y toma de decisiones & 8. Orientación al servicio \\
\hline 9. Escucha activa & 9. Negociación \\
\hline 10. Creatividad & 10. Flexibilidad cognitiva \\
\hline
\end{tabular}

En el ámbito tecnológico, serán necesarios más profesionales conocedores de las TIC y de la mecatrónica, científicos y analistas de datos, estadísticos y matemáticos, capaces de gestionar y poner en valor el Big Data. También serán de vital importancia los expertos en la interacción hombre máquina, y en la gestión de las nuevas formas de organización consecuencia del cambio tecnológico asociado a la digitalización. La referencia [17] expone el resumen que se muestra en la tabla 2 . 
Tabla 2 Conocimientos y habilidades en la fábrica del futuro.

\begin{tabular}{|c|c|c|c|}
\hline & Tiene que (Must)... & Debe (Should)... & Puede(Could)... \\
\hline \multirow{5}{*}{ 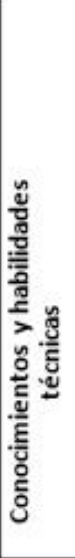 } & $\begin{array}{l}\text { Conocimientos y habilidades en } \\
\text { tecnologías de la información y } \\
\text { las comunicaciones (TIC) }\end{array}$ & Gestión del conocimiento(KM) & $\begin{array}{l}\text { Habilidades de programación } \\
\text { informática }\end{array}$ \\
\hline & $\begin{array}{l}\text { Proceso y análisis de datos e } \\
\text { información }\end{array}$ & $\begin{array}{l}\text { Conocimientos } \\
\text { interdisciplinarios genéricos } \\
\text { sobre tecnologias y } \\
\text { organizaciones }\end{array}$ & $\begin{array}{l}\text { Conocimiento especializado de } \\
\text { tecnologias: VR, AR, AM, IA, } \\
\text { loT, sensores, robótica, etc. }\end{array}$ \\
\hline & Conocimientos sobre estadistica & \multirow{2}{*}{$\begin{array}{l}\text { Conocimiento especifico de las } \\
\text { actividades y procesos de } \\
\text { fabricación }\end{array}$} & \multirow{2}{*}{$\begin{array}{l}\text { Concienciación acerca de la } \\
\text { ergonomía }\end{array}$} \\
\hline & $\begin{array}{l}\text { Compresión de las } \\
\text { organizaciones y los procesos }\end{array}$ & & \\
\hline & $\begin{array}{l}\text { Capacidad para interactuar con } \\
\text { los interfaces modernas: HMI, } \\
\text { HRI }\end{array}$ & $\begin{array}{l}\text { Concienciación sobre seguridad } \\
\text { y protección de datos }\end{array}$ & $\begin{array}{l}\text { Comprender las cuestiones } \\
\text { legales }\end{array}$ \\
\hline \multirow{5}{*}{ 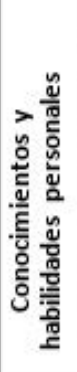 } & $\begin{array}{l}\text { Autogestión y gestión del } \\
\text { tiempo }\end{array}$ & $\begin{array}{l}\text { Confianza en las nuevas } \\
\text { tecnologías }\end{array}$ & \\
\hline & $\begin{array}{l}\text { Flexibilidad y adaptación al } \\
\text { cambio }\end{array}$ & $\begin{array}{l}\text { Mentalidad de mejora continua } \\
\text { y formación continua }\end{array}$ & \\
\hline & $\begin{array}{l}\text { Habilidades para el trabajo en } \\
\text { equipo }\end{array}$ & & \\
\hline & Habilidades sociales & & \\
\hline & Habilidades Comunicativas & & \\
\hline
\end{tabular}

Los nuevos planteamientos multidisciplinares son más que la simple suma de las partes (por ejemplo la mecatrónica). Esto afecta a las propias estructuras organizativas de las universidades. Por otra parte se imponen enfoques transversales, como la formación por proyectos, frente al enfoque clásico de disciplinas verticales aisladas.

Sub-reto 4: adopción de los nuevos métodos y tecnologías de formación.

Los métodos y tecnologías educativos modernos pueden mejorar los resultados del aprendizaje y hacer que éste sea interactivo, efectivo y atractivo para las nuevas generaciones. Formar en industria 4.0 utilizando sus mismas tecnologías.

Estamos pues ante un cambio en poliédrico que afecta a diversas dimensiones de la actividad empresarial y educativa, que comportará cambios a todos los niveles y especialmente en la educación superior. Iniciativas como "Universities of the Future" [18] intentan avanzarse a esta realidad y ser quienes generen un cambio paradigmático en la forma en que las instituciones de educación superior, empresas, industrias y administraciones públicas cooperan en el marco de Industria 4.0. Este informe presenta puntos de referencia sobre las mejores prácticas educativas en este campo, y discute sus posibles implicaciones sobre cómo organizar la educación en el futuro.

También las asociaciones y colegios profesionales están desarrollando una intensa labor en esta misma dirección, nariedad y de la integración de conocimientos anteriormente aislados. 
atendiendo las necesidades de colectivos como el de los ingenieros, que sienten la urgencia de adaptarse a esta nueva realidad ya desde el ejercicio activo de la profesión [19].

\section{Conclusiones}

La digitalización no es un objetivo en sí misma, sino un posibilitador de cambios en nuestras industrias, algunos disruptivos y otros simplemente evolutivos. La industria 4.0 comporta la aplicación innovadora de multitud de nuevas tecnologías pero de nuevo no es este el centro de la cuestión. La transformación hacia un nuevo paradigma industrial viene asociada a toda una serie de cambios sociales y económicos que discurren en paralelo.

Ello supone grandes retos no solo para las empresas sino también para toda la cadena de valor industrial, incluidas las instituciones de educación técnica y superior que deberán facilitar la transformación de la fuerza laboral existente y la creación de nuevos perfiles profesionales adaptados a esta nueva realidad.

Las empresas deben abordar la innovación de forma decidida y como parte de sus procesos estratégicos. No moverse es quedarse atrás en un entrono de cambio acelerado.

Las instituciones educativas por su parte no suelen ser ágiles ni fáciles de transformar, y existe el riesgo de que la velocidad del cambio externo supere su capacidad de adaptación. Es momento de impulsar una profunda reflexión sobre el tema orientada a la acción. Este artículo es una modesta aportación al debate que ahora simplemente empieza.

\section{Referencias}

CARLBERG, M., KREUTZER, S., MOELLER, C., SMIT, J. (2016), Industry 4.0 Analytical Study. Ed Policy Department A: Economic and Scientific Policy. European Parliament.

HELBIG, J., KEGERMANN, H., WAHLSTER, W., et al. (2013). Recommendations for implementing the strategic initiative INDUSTRIE 4.0. Ed. ACATECH Na-tional Academy of Science and Engineering.

INDRA，B. SANTANDER, TELEFÓNICA. (2015). La Transformación digital de la Industria española. Ed. Ministerio de Industria, Energía y Turismo de España.

BAUER H., et al (2015). Industry 4.0 How to navigate digitization of the manufacturing sector. Ed. McKinsey Digital.
LORENZ, M., RÜBMANN, M., et al. (2015). Industry 4.0. The Future of Productivity and Growth in Manufacturing Industries. Ed. The Boston Consulting Group.

CHUI, M., McCARTHY, B.(2018). An Executive's Guide to AI, Ed. McKinsey\&Company.

GEISSBAUER, R., VEDSØ, J., SCHRAUF, S. (2016). A Strategist's Guide to Industry 4.0.

VDMA Industrie 4.0 Forum Document (04/2016). Industrie 4.0 in practice -Solutions for industrial applications. Ed. VDMA.

IMPULS Foundation. Industry 4.0 Readiness. Online SelfCheck for Businesses.(2015). Ed: IMPULS Foundation of the German Engineering Federation (VDMA).

KEEBLE, J., LACY, P., MCNAMARA, R. (2014). Circular Advantage. Innovative Business Models and Technologies to Create Value in a World without Limits to Growth.Ed. ACCENTURE Strategy.

HUXTABLE, J., SCHAEFER, D.(2016). On Servitization of the Manufacturing Industry in the UK. Ed: ScienceDirect.

SCHMITZ, C., TSCHIESNER, A., ET AL. (2019). Industry 4.0. Capturing value at scale in discrete manufacturing Ed. McKinsey\&Company.

COSKUN, S., GENÇAY, E., KAYIKCI, Y. (2019), Adapting Engineering Education to Industry 4.0 Vision. Ed. MDPI

BJÖRKLUND, T., et al. (2019) Best practices in education from industry 4.0 perspective. Ed. UNIVERSITIESOF-THEFUTURE PROJECT.

BESTER A., SACKEY, S.M., (2016). Industrial Engineering Curriculum in Industry 4.0 in a South African Context. Ed: South African Journal of Industrial Engineering December 2016 Vol 27(4), pp 101-114.

RATCHEVA, V., TILL, A.L., ZAHIDI, S., (2016). The Future of Jobs. Employment, Skills and Workforce Strategy for the Fourth Industrial Revolution. Ed. World Economic Forum. 
GEHRKE, L., KÜHN, A., MOORE, P., RULE, D.,et al. (2015). A Discussion of Qualifications and Skills in the Factory of the Future, Ed. VDI \& ASME.

CLAVERT, M., et al.(2019) Industry 4.0 Implications for higher Education Institutions. Ed. UNIVERSITIESOF-THEFUTURE PROJECT.

J. AYZA, et al (2016). Industria 4.0, Status Report. Ed. Comissió Indústria 4.0, Enginyers de Catalunya. 


\section{ANEXO: Glosario de términos relacio- nados con la Industria 4.0}

AR (realidad aumentada): Tecnologías que permiten la superposición en tiempo real de objetos virtuales tridimensionales sobre un entorno físico existente de forma interactiva.

AI (Inteligencia artificial): Algoritmos que permiten realizar tareas consideradas hasta ahora propias de la inteligencia humana.

AM (Fabricación aditiva): Fabricación de objetos por aportación de material, generalmente creando capas sucesivas, a partir de sus modelos virtuales tridimensionales.

Big data: Volumen masivo de datos estructurados y no estructurados cuyo proceso resulta difícil utilizando técnicas convencionales de bases de datos y software.

Cloud (Nube): Sistema que permite acceder a recursos de TI a través de INTERNET y bajo demanda. Los recursos básicos disponibles son computación, almacenamiento y redes.

DL (Aprendizaje profundo): es un apartado del aprendizaje automático basado en redes capaces de aprender sin supervisión a partir de datos no estructurados o no etiquetados.

ERP (Sistema de Planificación de Recursos Empresariales): es un conjunto de sistemas de información que permite la integración de las operaciones empresariales:
HMI (Interfaz hombre-máquina): Interfaz de usuario que se usa para facilitar la interacción entre humanos y máquinas.

HRI (Interacción hombre-robot): es el estudio de las interacciones entre humanos y robots.

IIoT (Internet de las cosas industrial): red de objetos físicos conectados a Internet y las plataformas de análisis que procesan los datos que generan.

MES (Sistemas de ejecución de fabricación): Sistemas de información que permiten seguir en tiempo real y documentar la transformación de materias primas en productos terminados, ayudando a la toma de decisiones y optimización de las condiciones de producción.

ML (Aprendizaje automático): Algoritmos y modelos estadísticos que permiten realizar una tarea específica sin utilizar instrucciones explícitas, basándose en patrones e inferencias.

PLM (Gestión del Ciclo de Vida del Producto): Sistemas que permiten gestionar toda la información relacionada con el producto desde su diseño y desarrollo hasta su eliminación.

Servitización: Técnicas para aumentar el valor de los productos mediante la prestación de servicios adicionales que complementan su uso, función, o aplicación.

VR (Realidad virtual): Sistema informático que genera en tiempo real representaciones virtuales tridimensionales de la realidad, con las que el usuario puede interaccionar de forma inmersiva. 